\title{
Erratum to: Entrepreneurial Wisdom
}

\author{
Thomas Walker
}

\section{Erratum to:}

(C) Springer International Publishing Switzerland 2016

A. Habisch, R. Schmidpeter (eds.), Cultural Roots of Sustainable Management, CSR, Sustainability, Ethics \& Governance, DOI 10.1007/978-3-319-28287-9_13

\section{DOI 10.1007/978-3-319-28287-9_16}

The captions for Fig. 1, Fig. 2 and Fig. 3 were missing in the Chapter titled

"Entrepreneurial Wisdom". The correct captions are given below:

Fig. 1 The maturity model of corporate resilience

Fig. 2 The two dimensional St. Gallner management-model

Fig. 3 The integrative management approach

In pages 173,175 and 177 placeholders for the captions have been included in the published chapter. These placeholders have been removed and replaced by the correct captions.

The online version of updated original chapter can be found under DOI 10.1007/978-3-319-28287-9_13 